\title{
Circunscripción taxonómica de Schinus pilifera (Anacardiaceae) y características reproductivas de una población de La Calderilla, Salta, Argentina
}

Taxonomic circumscription of Schinus pilifera (Anacardiaceae) and reproductive characteristics of a population from La Calderilla, Salta, Argentina

Benci Arqued, José G.; Mariela Fabbroni; Emanuel Fuentes; Miriam N. Gil; Johana Villada; Gustavo Araya; Víctor H. Aquino; María M. Alemán; Evangelina C. Lozano; María A. Zapater*

Facultad de Ciencias Naturales y Consejo de Investigación, Universidad Nacional de Salta (U.N.Sa), Avda. Bolivia 5150, (4400) Salta, Argentina.

* Autor corresponsal: aliciazapater@yahoo.com.ar

\section{RESUMEN}

Schinus pilifera es un arbusto nativo del noroeste y centro de Argentina con amplia distribución en siete provincias y también en el sur de Bolivia. Es una especie muy polimórfica que ha sido dividida intra-específicamente en tres variedades: var. piliferus, var. boliviensis y var. cabrerae en base a caracteres de pubescencia y glabrescencia de sus hojas. No se encontró información sobre la variación morfológica, el sistema sexual y reproductivo de la especie. Los objetivos fueron obtener información morfológica general, del sistema reproductivo y analizar la validez de los taxones intraespecíficos. Se estudiaron colecciones de los herbarios LIL y MCNS además de herbarios virtuales BM, GH, MO y NY. Se realizaron nuevas colecciones en diferentes ambientes de las provincias de Salta y Tucumán a los fines de conocer y analizar la variación entre las poblaciones. Se estudiaron varios aspectos morfológicos y reproductivos de una población en La Calderilla, Departamento La Caldera (Salta) instalada en un antiguo lecho del río La Caldera a $1300 \mathrm{~m}$ snm. Se concluyó que los

\footnotetext{
Ref. bibliográfica: Benci Arqued, J. G.; Fabbroni, M.; Fuentes, E.; Gil, M. N.; Villada, J.; Araya, G.; Aquino, V. H.; Alemán, M. M.; Lozano, E. C.; Zapater, M. A. 2019. “Circunscripción taxonómica de Schinus pilifera (Anacardiaceae) y características reproductivas de una población de La Calderilla, Salta, Argentina". Lilloa 56 (2): 18-36. Fundación Miguel Lillo, Tucumán, Argentina. D.O.I.: doi.org/10.30550/j.lil/2019.56.2/2 Recibido: 21/12/18 - Aceptado: 15/08/19 > URL de la revista: http://lilloa.lillo.org.ar 
caracteres que definían a las variedades resultaron confusos por lo que fueron invalidadas y pasaron a la sinonimia. La especie es dioica. La polinización es ambófila.

Palabras clave - Morfología, molle, nomenclatura, sistema reproductivo.

\section{ABSTRACT}

Schinus pilifera is a native shrub from northern and central Argentina with a wide distribution in seven provinces also found in southern Bolivia. It a very polymorphic species that has been split into three intra-specific varieties: var. piliferus, var. boliviensis and var. cabrerae based on leaves pubescence and glabrescence characteristics. No information was found about the morphological variation, or sexual and reproductive systems of this species. The objectives were to obtain general morphological information on the reproductive system and analyze the validity of the intraspecific taxa. LIL and MCNS herbaria collections were studied, as well as the virtual ones from $\mathrm{BM}, \mathrm{GH}, \mathrm{MO}$ and $\mathrm{NY}$ herbaria. New collections were made in different environments of Salta and Tucumán to recognize and analyze the variation among populations. Several morphological and reproductive aspects were studied of a population from La Calderilla, Department La Caldera (Salta), located in an old bed of La Caldera River at $1300 \mathrm{~m}$ asl. It was concluded that the characteristics defining the varieties were confusing, reasonwhy they were invalidated and passed to the synonymy. The species is dioecious. The pollination is ambophilc.

Keywords - Morphology, molle, nomenclature, reproductive system.

\section{INTRODUCCIÓN}

El género Schinus L. es el de mayor magnitud de la Familia Anacardiaceae por el elevado número de especies. Fue dividido intra-genéricamente por Barkley (1944) en dos Subgéneros: Duvaua (Kunth) F. A. Barkley con hojas simples, inflorescencias en pseudoracimos y tallos espinescentes o no y Euschinus F. A. Barkley con hojas compuestas, inflorescencias mayormente panículas y tallos no espinescentes. En el Subgénero Duvaua se diferenció la Sección Euduvaua F. A. Barkley con ramas espinescentes y la Sección Pseudoduvaua F. A. Barkley con ramas no espinescentes. Schinus pilifera I. M. Johnston, motivo de este trabajo, fue incluido en el Subgen. Duvaua Sect. Eduvaua por poseer hojas simples y ramas espinescentes.

En el género Schinus se encontró una amplia variación de opiniones respecto al sistema sexual. Para (Cabrera, 1938; Muñoz, 2000) son arbustos poligamo-dioicos; Barkley (1957) solo describe flores estaminadas y pistiladas; según Juárez de Varela y Novara (2007) son arbustos dioicos o polígamo-dioicos; para Silva-Luz et al. (2018) son arbustos dioicos. De S. pilifera ningún autor emitió opinión sobre el sistema sexual, incluido Silva-Luz et al. (2018) que consideró a todos los Schinus como dioicos. Sin embargo estudios recientes realizados en Schinus areira L. sobre 
una población cultivada en la Universidad Nacional de Salta, mostraron que si bien la mayoría de los árboles eran dioicos, un número menor eran polígamo-monoicos (Zapater et al., 2018).

Recientemente, Silva-Luz et al. (2018) consideraron a Schinus un género con una elevada inconsistencia morfológica en la clasificación intra-genérica y la necesidad de modificación en la delimitación de las especies. A partir de esta base investigaron las relaciones filogenéticas de 44 taxones y muestrearon 122 especímenes concluyendo, a pesar de la aparente monofilia de Schinus, que el Subgénero Euschinus y las Secciones del Subgénero Duvaua son polifiléticas. De las relaciones filogénéticas emergentes de este estudio surgieron ocho linajes, si bien las relaciones entre las especies de algunos clados fueron poco claras. Aun así, varios caracteres morfológicos y anátomo-foliares resultaron muy adecuados para caracterizar los linajes. Sobre esta base se generó una clasificación de Schinus en ocho Secciones, considerando una combinación de caracteres morfológicos y anatómicos asociados con la distribución geográfica. Así, la Sect. Pilifera Silva-Luz \& J. D. Mitch. quedó integrada por unas nueve especies, aunque las relaciones entre ellas no fueron esclarecidas; además se detectó la existencia de especies crípticas o desconocidas. Por ello, los autores plantearon la necesidad de una revisión taxonómica para la identificación de caracteres nuevos que podrían ser utilizados en la delimitación de las especies, sobre la base de considerar a $S$. pilifera como un complejo (Silva-Luz et al., 2018).

Las especies de la Sect. Pilifera habitan en un amplio rango de tipos de vegetación, en los bosques xerofíticos deciduos y los arbustales del Monte y el Espinal principalmente en Argentina y también en la savana Uruguayense. Schinus johnstonii F. A. Barkley incluida en esta misma Sección, es la especie más austral con densas poblaciones desde el Monte hasta la Estepa patagónica (Silva-Luz et al., 2018).

Las características diagnósticas mencionadas por Silva-Luz et al. (2018), para la Sect. Pilifera fueron: arbustos generalmente armados con ramas de hojas simples, a menudo con hojas diferentes en forma y tamaño a las adultas; las inflorescencias en pseudoracimos, tirsoides y panículas; las flores pentámeras con pétalos más cortos que los estambres y los frutos globosos, rosados a purpúreos. El estudio anatómico de las hojas reveló un mesófilo iso-bilateral, tejido vascular del pecíolo y nervio medio dispuesto en haces vasculares de forma creciente y pecíolo con parénquima homogéneo. Los taxones incluidos fueron: S. bumelioides I. M. Johnst., S. fasciculata (Griseb.) I. M. Johnst., S. johnstonii F. A. Barkley, S. pearcei Engl., S. pilifera I. M. Johnst., con tres variedades: $S$. pilifera var. boliviensis (F. A. Barkley) F. A. Barkley y $S$. pilifera var. cabrerae (F. A. Barkley) F. A. Barkley y la variedad típica, S. praecox (Griseb.) Speg., S. sinuata (Griseb.) Engl. y S. uruguayensis (F. A. Barkley) Silva-Luz.

Schinus pilifera fue descripta por Johnston (1938) para Bolivia y Argentina, designándose como tipo un espécimen de S. Venturi proveniente de Alemania (Departamento Guachipas, Salta) (GH, sub nom. Schinus piliferus). En 1944, Barkley describió dos taxones afines: Schinus cabrerae F. A. Barkley y Schinus fasciculatus (Griseb.) I. M. Johnston var. boliviensis F. A. Barkley, a los que posteriormente transfirió, en 1957, a $S$. piliferus con rango varietal, generando de este modo tres variedades: var. piliferus, var. cabrerae (F. A. Barkley) F. A. Barkley y var. boliviensis (F. A. Barkley) F. A. Barkley, diferenciadas por la distribución de la pubescencia en las hojas. 
Por otra parte, en la principal bibliografía genérica (Barkley, 1957; Muñoz, 2000), la diferenciación de $S$. pilifera respecto a especies afines se basa exclusivamente en caracteres foliares (forma de lámina, margen, ápice y distribución de la pubescencia/glabrescencia para las variedades), lo que resulta confuso para la identificación de los individuos a campo y de los ejemplares en herbario.

En el año 2000, Muñoz en Flora Fanerogámica Argentina mantuvo a S. pilifera con sus tres variedades (citada como $S$. piliferus). Por su parte, Juárez de Varela y Novara (2007), en Flora del Valle de Lerma, comentaron que no pudieron diferenciar las variedades potencialmente existentes entre los materiales estudiados para esa área de la provincia de Salta. A la fecha se mantienen vigentes las tres variedades de $S$. pilifera tanto en la Flora del Cono Sur (www.darwin.edu.ar) como en Flora Argentina (www.floraargentina.edu.ar), según la base de datos disponibles on line.

Respecto a la sexualidad, como se mencionó anteriormente, no hay definiciones sobre la sexualidad de $S$. pilifera por ninguno de los autores consultados (Barkley, 1957; Muñoz, 2000; Silva-Luz et al., 2018). Tampoco se encontró información acerca de su biología floral y sistema reproductivo; además, los caracteres morfológicos reproductivos no fueron utilizados para la diferenciación específica.

El fruto drupa del género Schinus de las Anacardiaceae en general, según Baskin, Baskin, Li (2000), posee semilla indiferenciada y protegida por el fruto duro (endocarpo) formado por tres capas impermeables de esclereidas en empalizada, que determinan dormición física. No se encontró en la bibliografía consultada estudios particularizados sobre la morfología y anatomía del fruto drupa de $S$. pilifera.

Por lo expuesto, los objetivos de la presente investigación fueron 1) revisar y seleccionar los caracteres morfológicos vegetativos (de planta adulta y plántula) y reproductivos, con mayor peso para la delimitación taxonómica de $S$. pilifera; 2) analizar la validez de los caracteres diagnóstico de las variedades; 3) determinar la sexualidad y el sistema reproductivo estudiando una población de la localidad de La Calderilla (Salta-Argentina); 4) analizar los caracteres morfo-anatómicos del fruto y su relación con la identificación taxonómica.

\section{MATERIALES Y MÉTODOS}

\section{Análisis taxonómico}

Se estudiaron los ejemplares de las colecciones de los siguientes herbarios de Argentina (citados por sus acrónimos) LIL y MCNS e imágenes virtuales consultados «on line» de los herbarios $\mathrm{BM}, \mathrm{GH}, \mathrm{MO}$ y NY, los que se describieron en forma completa, analizando los caracteres específicos y varietales. A campo, se trabajó durante el período 2016-2017 en el estudio de la biología reproductiva de una población nativa de S. pilifera como especie dominante de una comunidad de Chaco serrano constituida también por Scutia buxifolia Reissek. (coronillo), Vachellia caven (Molina) Seigler \& Ebinger (churqui), Vachellia aroma (Gillies ex Hook. \& Arn.) Seigler \& Ebinger (tusca), Allophylus edulis (A. St.-Hill., A. Juss. \& Cambess.) Hieron. ex Niederl. (chal chal), Celtis iguanea (Jacq.) Sarg. (tala gateador), Tecoma stans (L.) Juss. ex Kunth. (guarán 
amarillo) y Xylosma pubescens Griseb. (espina corona). La comunidad se extiende en un sector marginal del río La Caldera en la localidad de La Calderilla (Departamento La Caldera, Salta), sobre un antiguo cauce con sustrato areno-pedregoso (Fig. 1A). Se estudiaron caracteres vegetativos de ramas y hojas en diferentes estadios etarios; también reproductivos de inflorescencias y flores en diferentes estadios fenológicos, los que fueron contrastados con otras poblaciones estudiadas de Salta y Tucumán. Para determinar las características florales (cualitativas y cuantitativas) se colectaron 30 flores (15 pistiladas y 15 estaminadas) en antesis provenientes de individuos diferentes tomadas al azar. Diez flores fueron diseccionadas bajo lupa y cada una de sus piezas (longitud y ancho de sépalos y pétalos, longitud y ancho de estambres, longitud y ancho de ovario, estilo y estigmas, longitud y ancho de estaminodios y longitud y ancho de pistilodios); también de frutos (diámetros) que fueron medidos en milímetros con un calibre digital. Las otras 10 flores fueron fijadas en FAA para su posterior análisis más detallado en el microscopio electrónico de barrido (MEB; JEOL JSM-6480 LV). Para ello las flores fueron tratadas con las técnicas convencionales propias de este tipo de estudio (D’Ambrogio de Argüeso, 1986).

\section{Biología floral y reproductiva}

Debido a la variación de opiniones entre autores acerca de la sexualidad genérica, se decidió experimentar con árboles de sexualidad aparentemente separada. A principios de setiembre del 2016, se aplicaron dos tratamientos considerados primarios y generalmente utilizados en especies con flores perfectas, en 10 individuos de la población (cinco estaminados y cinco pistilados) seleccionados al azar, considerados coetáneos por compartir el mismo porte (altura total hasta $5 \mathrm{~m}$ y fustes de $10-15 \mathrm{~cm}$ de Diámetro a la Altura de Pecho, DAP). Los tratamientos fueron Autopolinización Espontánea (APE) y Polinización libre (PL), que contribuirán a una rápida e indirecta aproximación al conocimiento de la sexualidad de la población. Para APE se embolsaron cinco inflorescencias por árbol en estado de botón floral con bolsas de voile de alta densidad que impedía el paso de polen transportado por el viento, circundando la copa a nivel medio externo. Para PL, se marcaron en los mismos individuos con cintas plásticas otras cinco inflorescencias elegidas al azar en posiciones similares. Alcanzada fructificación a comienzos de noviembre-diciembre, se extrajeron las muestras embolsadas y libres, para efectuar los recuentos de flores producidas y número de frutos logrados. Se calculó el Éxito Reproductivo Relativo (ERR) de cada tratamiento a través de la relación $\left(\mathrm{N}^{\circ}\right.$ de frutos $/ \mathrm{N}^{\circ}$ de flores $)$ x $\left(\mathrm{N}^{\circ}\right.$ de semillas $/ \mathrm{N}^{\circ}$ de óvulos) x 100 , sobre la base de que los frutos son uniseminados (Dafni, 1992).

La proporción P/O (Polen/Óvulo) se determinó en laboratorio a partir de 10 botones florales. Se extrajeron separadamente los estambres de cada ciclo (cortos y largos), se maceraron en $0,5 \mathrm{ml}$ de una solución de detergente, agua y alcohol, se homogeneizaron en vórtex; y luego se extrajo una pequeña alícuota que fue colocada en un hemocitómetro para el conteo de granos de polen. Obtenido el $\mathrm{P} / \mathrm{O}$, resultante del promedio de las 10 flores, se comparó con los valores índices de la tabla de Cru- 
den (1977) y de Dafni (1992), obteniendo la clasificación del sistema reproductivo correspondiente a la especie.

Tipo de Polinización.- En 2017, en el mes de octubre, con el objetivo de conocer el o los tipos de polinización (eófila, entomófila o ambófila) se realizó un nuevo embolsamiento con tul, tejido que permite el ingreso de granos de polen del exterior pero no a los polinizadores, en cinco inflorescencias de cinco árboles pistilados en el área de la población. Producida la fructificación se determinó el porcentaje de frutos producidos por inflorescencia y el promedio por árbol.

Morfología del fruto.- Para el estudio de los frutos se seleccionaron al azar 25 drupas maduras de 10 individuos de la misma población, en las que se registró el diámetro por la forma esférica de las drupas y el peso específico con exocarpo, empleando calibre digital y balanza de precisión. También se determinó el peso de 100 frutos con y sin exocarpo a los fines reproductivos en vivero. Se describió con LB y MEB la morfología del fruto y la semilla.

\section{RESULTADOS}

\section{Tratamiento taxonómico}

Schinus pilifera I. M. Johnston, J. Arnold Arb. 19: 256. 1938. TIPO: Argentina. Salta, Dpto. Guachipas, Alemanía, 1300 m snm, S. Venturi 9830, 27-XI-1929 (holotipo GH00049186!; isotipo BM000884833!). (Figs.1, 2, 3).

Schinus fasciculata (Griseb.) I. M. Johnst. var. boliviensis F.A. Barkley, Brittonia 5: 177. 1944. Schinus. pilifera I. M. Johnston var. boliviensis (F.A. Barkley) F.A. Barkley, Lilloa 28: 39. 1957. TIPO: Bolivia, Bolivia plateau, M. Bang 981, 1891 (holotipo MO-260108!).

Schinus cabrerae F.A. Barkley, Brittonia 5: 173. 1944. Schinus pilifera I. M. Johnston var. cabrerae (F.A. Barkley) F.A. Barkley, Lilloa 28: 38. 1957. TIPO: Argentina. Tucumán, Trancas, D. Rodríguez 1167, 10-XII-1913 (holotipo NY00050880!).

Arbusto perennifolio, a veces con aspecto arborescente y copa irregular de hasta $5 \mathrm{~m}$ alt. (Fig. 1B), diclino-dioico (determinado en este trabajo a partir del estudio de éxito reproductivo), aromático, corteza oscura longitudinalmente fisurada (Fig. 1C), con varios troncos basales de hasta $10-15 \mathrm{~cm}$ diám. Las ramas basales de la copa son de color gris claro, estriadas, rugosas; las de menor edad hasta de 2 y 3 años a menudo espinescentes, pardo-rojizas (Fig. 1D), con pubescencia corta, densa a semidensa, de tricomas muy cortos, blancos, erectos; con pequeñas lenticelas negras esparcidas. Las ramas juveniles de 1-2 años, verdosas y con tonalidades rojizas al aumentar en edad son densamente pubescentes. Sobre las ramas de 2-3 años, los individuos pistilados de diferentes edades desarrollan 1-6 ramas espinescentes floríferas 

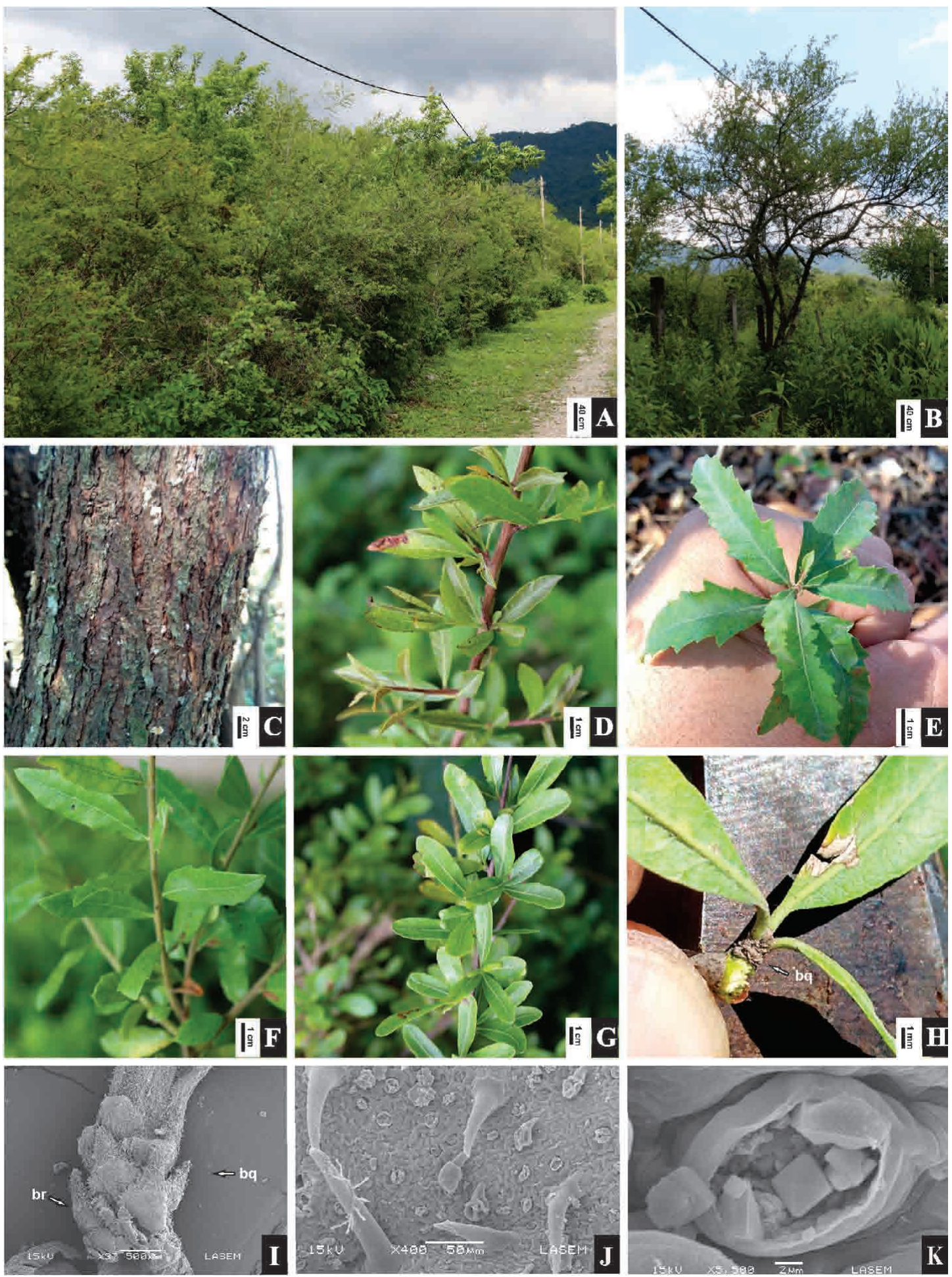

Fig. 1. Schinus pilifera. A) Vista de la población estudiada en La Calderilla, Salta. B) Vista de un individuo. C) Aspecto de la corteza. D) Ramas espinescentes pardo rojizas. E) Plántula de 90 días, hojas irregularmente aserradas. F) Rama de individuo adulto con hojas solitarias alternadas. G) Rama de individuo adulto con hojas fasciculadas. H) Braquiblasto con fascículo de hojas adultas. I) Detalle del braquiblasto y las brácteas (MEB). J) Epidermis foliar con diferentes tipos de tricomas e idioblastos (MEB). K) Detalle de un Idioblasto lisado con aparentes cristales de oxalato de Ca (MEB). Abreviaturas: bq, braquiblasto; br, bráctea. A-H: fotografías propias de los autores. I-K: fotografías al MEB por Carlos A. Gómez. 
de 0,9-9,5 cm con hasta 24 nudos (Fig. 2A); y los individuos estaminados hasta 13 ramas espinescentes floríferas de 2-4(-6) cm, con hasta 22 nudos (Fig. 2G). Sobre las ramas de 2-3 años también se forman agallas leñosas esféricas castañas, dispersas, muy características de la especie, que no llegan a afectar la función fotosintética ni la reproductiva (Fig. $3 \mathrm{H}$ ). Hojas simples con láminas de 2,5-4 x 0,5-1,5 cm, alternas (Fig. 1F) o fasciculadas (Fig. 1G), fascículos de 2-4 (-6) hojas semejantes en forma y tamaño dependiendo de la edad, si son de ramas apicales o basales y si la rama es florífera o no. Hojas fasciculadas dispuestas sobre braquiblastos delgados de 1,5-3 (-4) x 1,5-2 mm, amarronados, con entrenudos muy cortos y brácteas de 0,2-0,9 x 0,5-1 $\mathrm{mm}$, triangulares a deltoides con pubescencia densa; láminas obovadas y elípticas, a veces con 2-3 pares de dientes en la mitad superior con 1-3 $\mathrm{mm}$ de profundidad, discoloras, rugosas, con 10-15 nervios paralelos no perpendiculares formando ángulos con el nervio principal de $30-40^{\circ}$, base cuneada, ápice agudo, a veces mucronado o retuso, margen entero ligeramente revoluto con pequeñísimos pelos esparcidos; glabras a subglabras en el haz o con pubescencia generalizada y diversos tipos de tricomas (Fig. 1J), simples largos y agudos de $0,13 \times 0,02 \mathrm{~mm}$, aguijones de $0,04 \mathrm{x}$ $0,017 \mathrm{~mm}$ y tricomas glandulares generalmente pedicelados con una célula basal mediana de $0,012 \times 0,013 \mathrm{~mm}$ y una cabeza apical grande de $0,037 \times 0,023 \mathrm{~mm}$, que al $\mathrm{MO}$ se observa constituida por varias células; idioblastos con aparentes cristales de oxalato de calcio. Envés subglabro con márgenes y nervio principal pilosos, a veces con pilosidad generalizada y pubescencia glandular; pecíolos canaliculados de (0,5-) 1,5-7 mm, pubescentes a subglabros, con tricomas de 0,1-0,5 mm. Inflorescencia tirsoide de cimas dicasiales sésiles [Fig. 2 B (LB) y Fig. 2K (MEB)], de (0,2-) 0,6-4 cm, con eje principal terminado en flor, con 7-8 brácteas basales anchamente ovadas a deltoides de ca. 0,9 x 0,6 mm, largamente acuminadas, acumen de aproximadamente $0,55 \mathrm{~mm}$ densamente piloso con tricomas largos y flexuosos, dorso subglabro (Fig. 2L) (MEB); con hasta 24 flores en los tirsos pistilados y hasta 39 flores en los estaminados (Fig. $2 \mathrm{H}$ ), raquis anguloso, piloso con pubescencia hírtula corta, semidensa y con 1-3 flores por nudo; los dicasios se forman en la base de una bráctea semejante a la de los fascículos de hojas, de 0,5-1 $\mathrm{mm} \times$ x 0,3-0,5 mm. Flores pistiladas (Fig. 2C; LB; Fig. 2D; MEB) de 2,7-4,6 mm, cáliz de 0,8-1 x 0,7-0,8 mm, gamosépalo, pentámero, sépalos deltoides, ápice redondeado, borde irregularmente ciliado con tricomas glandulares o no; corola de 1,5-1,8 mm x 1-1,2 $\mathrm{mm}$ pentámera, pétalos blancos, ovados, ápice agudo a redondeado, deflexos en la mitad de su longitud, borde irregular, glabros; disco nectarífero carnoso rodeado de 10 estaminodios de 0,6-1 mm; ovario esférico de $2 \times 2 \mathrm{~mm}$, epidermis con tricomas glandulares pedicelados y sésiles (Fig. $2 \mathrm{E}, \mathrm{MEB}$ ), y con pelos cortos triangulares de diferentes tamaños, estilo corto de 0,29-0,47 mm y tres estigmas capitados (Fig. 2F, $M E B)$ de $0,3 \mathrm{~mm}$ diám., cabezas estigmáticas redondeadas, con membrana. Flores estaminadas (Fig. 2I, LB; Fig. 2J, MEB) de 2,3-2,8 mm, sépalos de 0,7-1,2 x 0,4-0,7 $\mathrm{mm}$, ovados, ápice redondeado, borde irregularmente ciliado; corola pentámera, pétalos de 1,5-3 x 1,2-1,6 mm, blancos, ovados, ápice redondeado, borde irregular; disco nectarífero carnoso interestaminal 10-lobulado, 10 estambres en 2 ciclos, el externo de 3,3-3,9 $\mathrm{mm}$ y el interno de 1,8-2,2 $\mathrm{mm}$; pistilodio sacciforme de $0,3 \times 0,2 \mathrm{~mm}$ con tres estilos y estigmas con hendidura central. Pedicelos (Fig. 2L, MEB) de $3 \mathrm{~mm}$ 

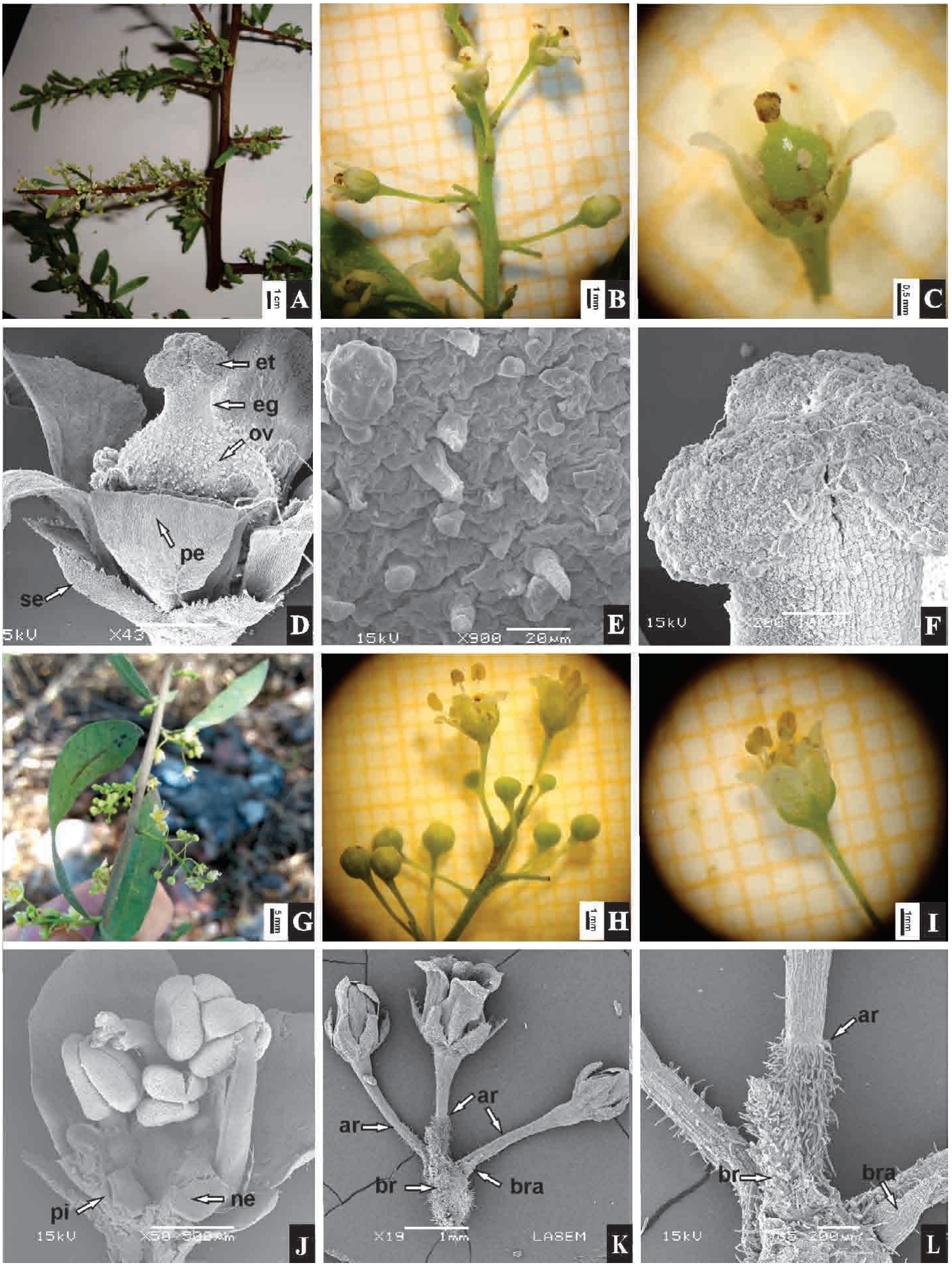

Fig. 2. Schinus pilifera. A) Rama espinescente florífera pistilada. B) Detalle de tirsoide dicasial pistilado (LB). C) Flor pistilada (LB). D) Flor pistilada al MEB. E) Aspecto de la epidermis del ovario con diferentes tipos de tricomas (MEB). F) Detalle del estigma tri-capitado con membrana gruesa (MEB). G) Rama florífera estaminada. H) Detalle del tirsoide dicasial estaminado (LB). I) Flor estaminada (LB). J) Flor estaminada al MEB. K) Dicasio sésil (MEB). L) Pedicelo floral articulado (MEB). Abreviaturas: ar, articulación del pedicelo; br, bráctea; bra, bractéola; bq, braquiblasto; eg, estilo; et, estigma; ne, nectario; ov, ovario; pe, pétalo; pi, pistilodio; se, sépalo. A -C, G-I: fotografías propias de los autores. D-F, J-L: fotografías al MEB por Carlos A. Gómez. 
articulados en la base, en la axila de una bracteóla basalmente ensanchada pedicelo apical de 2-2,2 $\mathrm{mm}$ por arriba del disco de abscisión, glabros o con pubescencia esparcida. Bractéola de 0,3 x 0,1-0,2 mm, triangular, bordes con tricomas pilosos y glandulares esparcidos. Pedicelo basal de 0,8-1 mm con pilosidad densa en la flor central del dicasio y con pubescencia esparcida en las flores laterales. Fruto drupa (Fig. 3C, LB) de 3,52 (3,40-4,67) mm diám., hasta aproximadamente $6 \mathrm{~mm}$, redondeada, morada a negra, exocarpo irregularmente piloso, con pelos de hasta $0,2 \mathrm{~mm}$ y tricomas glandulares pequeños, pedicelados, escasos. Los rebrotes y las plántulas a los 90 días, poseen hojas ovadas con tendencia hastiforme, haz verde oscuro brillante y envés verde claro opaco, nerviación craspedódroma simple muy pronunciada en el haz y envés, margen bi-aserrado (Moreno, 1984), ápice agudo, base anchamente cuneada (Fig. 1E). Al año los plantines con $106 \mathrm{~cm}$ long. en promedio desarrollan hojas principalmente ovadas y elípticas, con borde aserrado, raro bi-aserrado, ápice agudo y base pronunciadamente cuneada.

Nombre vernáculos.- Molle, molle blanco, cimarrón, molle criollo, molle pispito, molle tucumano, según Perea, Pedraza, Luceros (2007).

Distribución y hábitat.- Predominantemente, los ejemplares fueron colectados en ambientes de Chaco serrano, Chaco occidental y del Monte y en cercanías de cursos de agua o con freática elevada, sobre sustratos pedregosos y arenosos. Con menor frecuencia, se encontraron en Selva pedemontana de Yungas. Perea et al. (2007) lo citan para la ecoregión del Monte y en la región ecotonal con la Prepuna en la provincia de Catamarca desde los 500 hasta $2500 \mathrm{~m}$ snm., en áreas cercanas a los cursos de agua donde alcanza mayor desarrollo, aunque también fueron encontrados en claros o bajo algarrobos de gran porte. No se encontró variaciones morfológicas asociadas a lo diferentes ambientes biogeográficos.

Fenología.- En la población estudiada la floración se inició a mediados del mes de octubre y la fructificación plena se completó en la primera semana de diciembre. Suele producirse una segunda floración con formación de frutos maduros a comienzos de enero. En la localidad de Tapia (Tucumán) se encontró una población con inicio de floración en los pies estaminados a comienzos de febrero y con producción de frutos en mayo. En general, en la mayoría de los ejemplares estudiados en herbario y a campo, la fenología es aproximadamente coincidente a la del área de estudio en La Calderilla, Salta.

Material estudiado.- ARGENTINA. Prov. Catamarca, Dpto. Ambato, a $7 \mathrm{~km}$ de El Rodeo, 1.800 m snm, 12-I-1941(fr.), Arague 600 (LIL). Dpto. Capayán, Villa Santo Domingo 11-I-1947(fr.), Risso 870 (LIL). Dpto. El Alto, camino de Alijilán a El Alto, 670 m snm, 16-XI-1972(fl.), Menéndez Sevillano et al. 9333 (MCNS). Dpto. Paclín, cuesta del Totoral, pasando $10 \mathrm{~km}$ del Durazno, $1000 \mathrm{~m}$ snm, 14-XI-1972(fl., fr.), Legname et al. 9494 (MCNS). Dpto. Santa María, Palo Seco, 17-II-1948(fl.), Reales 934 (LIL). Prov. Córdoba, Dpto. Colón, río Carapé, 729 m snm, 23-XII-1947(fl., fr.), Meyer 13644 (LIL). Dpto. Punilla, La Falda, 26-XI-1929(fl.), Palacios 4291 (LIL). 

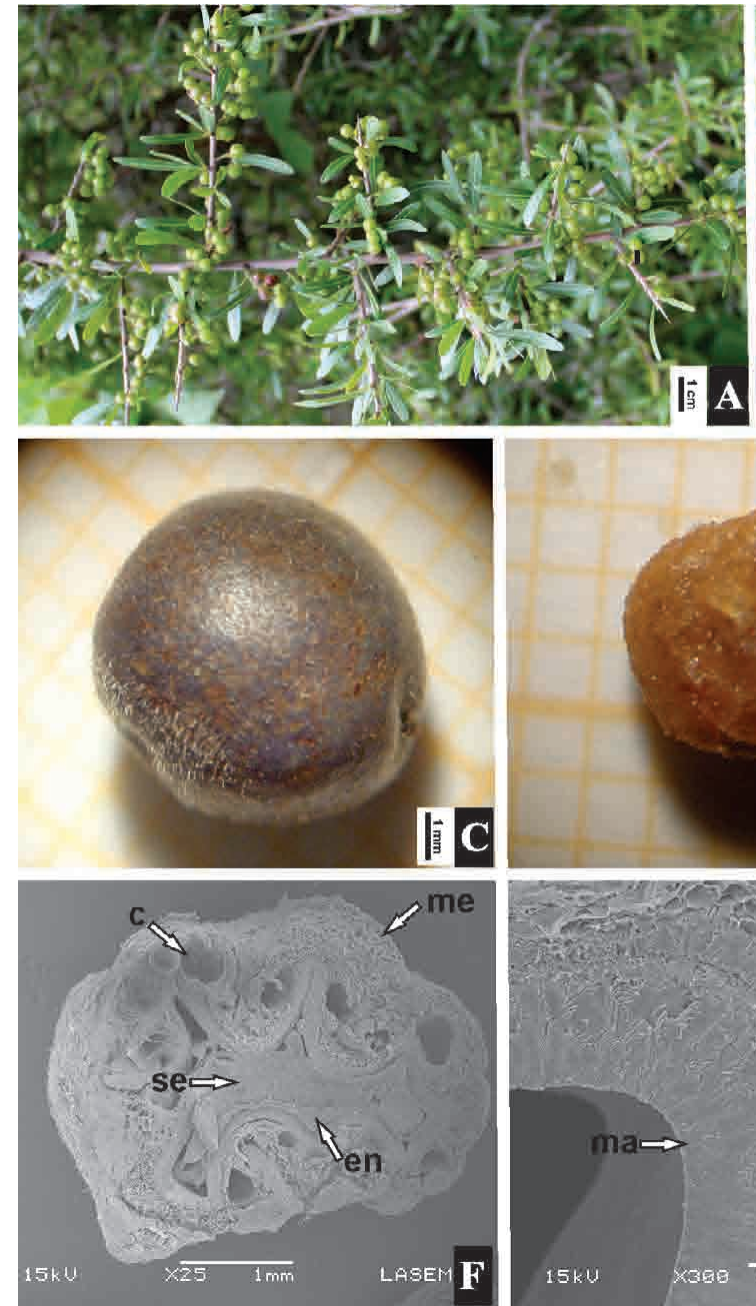
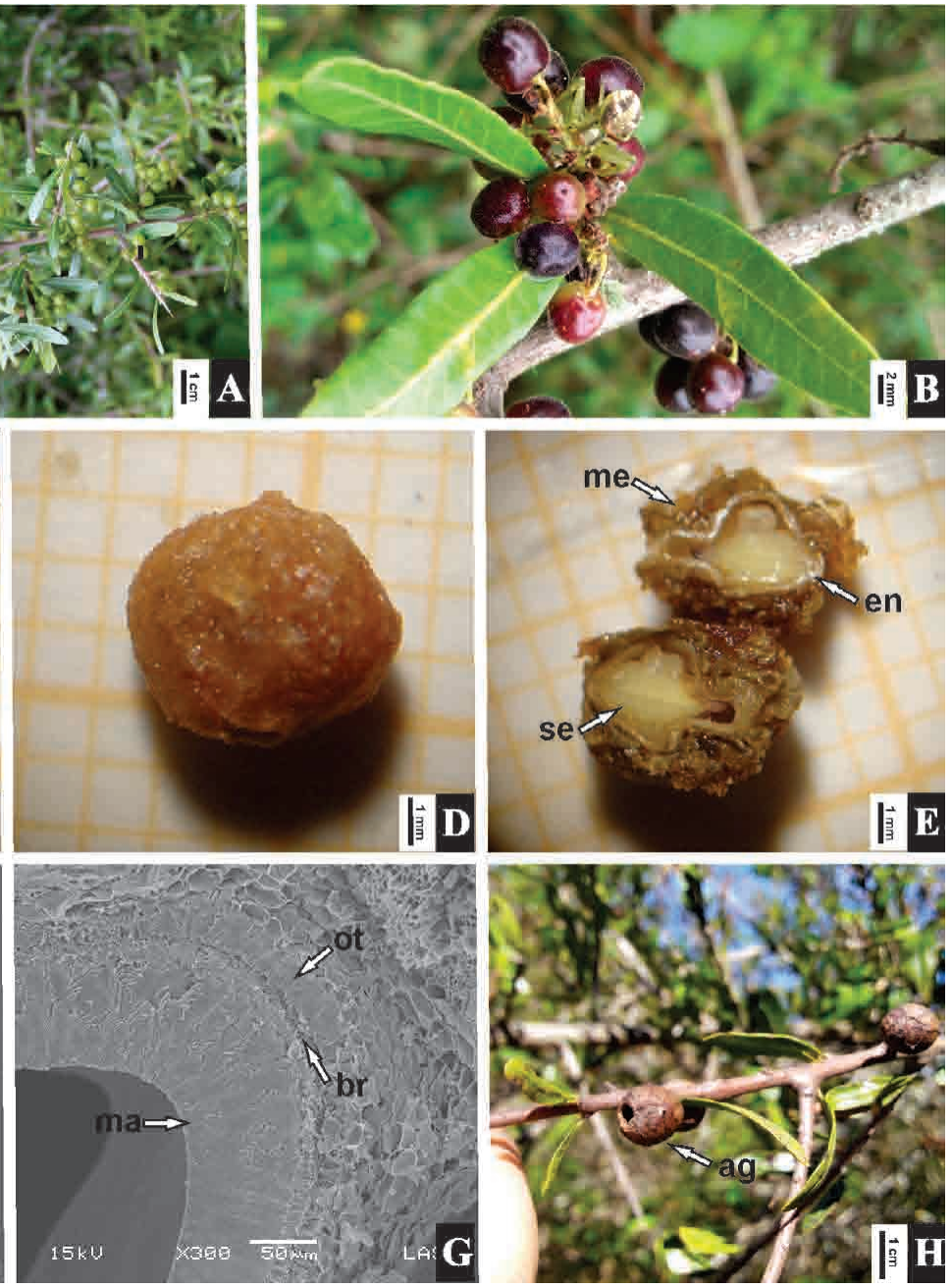

Fig. 3. Schinus pilifera. A) Rama con frutos inmaduros. B) Rama con frutos maduros. C) Drupa pubescente (LB). D) Fruto sin exocarpo, con mesocarpo resinoso (LB). E) Corte transversal del fruto (LB). F) Transcorte de fruto al MEB. G) Detalle de las tres capas de esclereidas del endocarpo (MEB). H) Agalla lignificada. Abreviaturas: ag, agalla; br, empalizada de braquiesclereidas; c, canales resiníferos; en, endocarpo; ma, empalizada de macroesclereidas; me, mesocarpo; ot, empalizada de osteoesclereidas; se, semilla. A-E, H, fotografías propias de los autores. F-G, fotos al MEB por Carlos A. Gómez.

Prov. Jujuy, Dpto. Tumbaya, Estación Volcán, 2100 m snm, 20-I-1927(fl.), Castillon 316 (LIL). Prov. La Rioja, Dpto. Chamical, Quebrada de Olta, Los Talas, 29-II1940(fl.), Castellano s.n. (LIL 33735). Prov. Salta, Dpto. Anta, Ceibalito, 2-X-1977(fl.), Saravia Toledo 6098 (LIL); Parque Nacional El Rey, Campo Santa Elena, 4-II-1984(fl., fr.), Chalukian 1911 (MCNS), Parque Nacional El Rey 10-X-1987; Vinal Pozo, 3-VI1947, Luna 212 (MCNS). Dpto. Cafayate, margen derecha del rio Lorohuasi, 1600 m snm, 12-V-2017(fr.), Zapater \& Benci 5315 (MCNS); margen izquierdo del rio Chuscha a $50 \mathrm{~m}$ de la Ruta Nac. 68, $1.600 \mathrm{~m}$ snm, 12-X-2017(fl.), Zapater $\mathcal{E}$ Benci 5517 (MCNS); Cafayate, $1650 \mathrm{~m} \mathrm{snm}$, 14-II-1951(fl.), Hayward s.n (LIL); bosques de algarrobo, 4-V-1989(fr.), Karlsson 62 (MCNS); rio Lorohuasi, margen derecha, 1638 m snm, 1-V-2017(fr.), Fabbroni 1475 (MCNS). Dpto. Capital, cercanías del Aeropuerto, frente al cementerio en campo abandonado y sobre alambrados, 21-X-2017(fl.), Zapater $\mathcal{E}$ Benci 5522 (MCNS); Campo militar, 5-X-2017(fl., fr.), Zapater $\mathcal{E}$ Fuentes 
5510 (MCNS); La Lagunilla, 6-III-1997(fr.), Fuárez de Varela E̋ Ochoa 2331 (MCNS); La Loma, 25-III-2017(fr.), Zapater E Gil 5297 (MCNS); La Pedrera, a 5-6 km al SE de la ciudad, ambiente de Chaco Serrano, 1200 m snm, 8-I-1998(fl.), Tolaba 991 (MCNS). Dpto. Cerrillos, Estación Experimental INTA Cerrillos, 1200 m snm, 18X-1996(fl.), Tolaba 746 (MCNS). Dpto. Chicoana, Chicoana, II-1942(fr.), Zabala 262 (MCNS). Dpto. La Caldera, Cuesta del Gallinato, entre rio La Caldera y abra del camino a Gral. Güemes, 1300-1500 m snm, 10-X-1987(fl.), Novara 6374 (MCNS); Gallinato, a 2,5 km del acceso a Ruta Nac. 9, 20-XII-2017(fr.), Zapater $\mathcal{E}$ Benci 5584 (MCNS); La Calderilla a $600 \mathrm{~m}$ de la Ruta Nac. 9 Km 1619, población a lo largo de las márgenes del rio La Caldera (estudiada), árbol 1 pistilado, a $20 \mathrm{~m}$ casa familia Benci, 1300 m snm, 21-X-2016(fl.), Zapater Ë Benci 5199; ibid, árbol 3 estaminado, a $5 \mathrm{~m}$ del camino vecinal, interior del bosque, población estudiada, $1300 \mathrm{~m} \mathrm{snm}$, 21-X-2016 (fl.), Zapater et al. 5201 (MCNS); ibid., de la misma población, individuo pistilado, $1300 \mathrm{~m}$ snm, 7-XII-2016(fr.), Zapater et al 5253 (MCNS); rio Mojotoro, en cercanías de vías del ferrocarril Gral. Belgrano, $4-5 \mathrm{~km}$ al NE del túnel ferroviario, 12-I-1998(fl.), Tolaba 1008 (MCNS); Vaqueros, a 1,3 km de Ruta Nac. 9, 2441,3'S 6525,1'W, $1330 \mathrm{~m}$ snm, 23-X-2010(fl.), Martinez et al. 2028 (MCNS), Vaqueros, a 1 km RN 9 S 24 41.5' W 65 25.1' a 100 m A ${ }^{\circ}$ Chaile, 23-XII-2010 (fl.), Chambi $\mathcal{E}$ Ramirez 270 (MCNS) Dpto. La Candelaria, rio Candelaria, 16-I-1964(fr.), Cuezzo et al. 3425 (LIL). Dpto. La Viña, Coronel Moldes, 1100 m snm, 31-I-1941(fl.), Meyer 3875 (LIL). Dpto. Guachipas, camino de acceso al pueblo a $100 \mathrm{~m}$ del rio Guachipas, sobre alambrados, 2-XI-2017(fl.), Zapater E Del Castillo 5534 (MCNS). Dpto. Rosario de la Frontera, cercanías del Hotel Termas, 1-XI-1965(fl.), Villa Carenzo 2696 (LIL); Dto. Rosario de Lerma, Campo Quijano, Dique Las Lomitas, 11-III-1998(fl., fr.), Novara et al. 483 (LIL); Campo Quijano 27-XIII-1993 (fl.), Garkus 1073 (MCNS). Dpto. Orán, Ruta La Estrella-Siete Aguas, Ruta Prov. 80, $7 \mathrm{~km}$ al W de La Estrella, 235'00"S 64²018'W, 750 m snm, 13-XII-2001(fr.), Mello E Silva-Luz 1908 (MCNS); rio Yacuí, 9-II-1925(fl.), Schreiter 3561 (LIL). Prov. Santiago del Estero, Dpto. La Banda, camino vecinal 25-XII-1946(fl.), O’Donell 4268 (LIL). Dpto. Giménez, El Charco, 16-IV-1930(fr.), Venturi 10113 (LIL). Dpto. Pellegrini, Estancia El Remate, 22-XII-1927(fl.), Venturi 5775 (LIL). Prov. Tucumán, Dpto. Burruyacu, cerro del Campo, 2000 m snm., I-1919(fr.), Bailetti 109 (LIL). Dpto. Capital, ciudad de Tucumán, 14-I-1940(fl., fr.), Meyer 3225 (LIL); Tapia, cercanías del camino de acceso a la ciudad, sobre bordes de alambrados 8-V-2017(fr.), Zapater $\mathcal{E}$ Del Castillo 5308 (MCNS). Dpto. Famaillá, rio Lules, 450 m snm, 14-X-1923(fl.), Venturi 2291 (LIL). Dpto. Leales, Leales, 12-II-1945(fl.), Krapovickas 1728 (LIL). Dpto. Rio Chico, La Cocha, 450 m snm, II-1919(fr.), Bailetti 538 (LIL). Dpto. Trancas, Camino a Tapia, 4-II-1970(fl., fr.), Cuezzo E Legname 4460 (LIL). BOLIVIA. Dpto. Santa Cruz, Prov. Valle Grande, Samaipata, 1100 m snm, 15-III-1920(fl.) Steinbach 3737 (LIL); Cerro de Alto Meirano, 2300 m snm, 21-XII-1921(fl.), Steinbach 6040 (LIL).

Consideraciones taxonómicas. - Las variedades actualmente aceptadas de $S$. pilifera se diferencian según Barkley (1957) y Muñoz (2000) en base a lo siguiente: la variedad típica por láminas glabras con pecíolos pubescentes y nervios con algunos tricomas en haz y envés; la variedad cabrerae por la pubescencia en pecíolos y lámina 
en general (nervios y espacios internervales) y la var. boliviensis por láminas glabras con pecíolos esparcidamente pubérulos. La variedad cabrerae fue citada para Salta, Tucumán y Catamarca hasta los $700 \mathrm{~m}$ snm., en tanto que la variedad típica y la variedad boliviensis con un área de distribución más amplia en Argentina (Catamarca, Córdoba, Jujuy, La Rioja, Salta, Santiago del Estero y Tucumán hasta los 2100 $\mathrm{m}$ snm). En Bolivia, la variedad boliviensis fue mencionada para los Departamentos de Cochabamba y Santa Cruz, mientras que la variedad cabrerae únicamente hasta Tarija; la variedad típica posee una mención para el territorio boliviano basado en una colección de Steinbach (1697, LIL, NY).

Durante el análisis de las colecciones existentes y las nuevas que se realizaron en este trabajo, fue muy complicado poder identificar con seguridad las variedades en base a las características de diferenciación planteadas en la bibliografía. La distribución de la pubescencia foliar es muy variable, a menudo en el mismo individuo o ejemplar, por lo cual en el extenso material estudiado que se citó previamente no se las pudo diferenciar.

Para realizar un análisis más detallado de las variedades, se seleccionaron 15 ejemplares de diferentes poblaciones y ambientes, con abundante material vegetativo foliar que permitiera analizar entre 10-20 hojas de cada uno. Las colecciones estudiadas fueron de Catamarca, Salta y Tucumán. De ellas, once no pudieron ser identificadas en ninguna de las tres variedades con base en la distribución de la pubescencia foliar. Las cuatro restantes se pudieron identificar como dos variedades con algunas inconsistencias, porque la bibliografía no especifica el tipo de pubescencia que se observó glandular y pilosa. Tampoco se pudo vincular la pubescencia con las condiciones ambientales de los lugares de colección. Los resultados de este estudio se muestran en Tabla 1 y 2 . Con base en este resultado, se consideran inválidas las variedades de $S$. pilifera, las que pasan a la sinonimia. Los caracteres morfológicos

Tabla 1. Resultados del análisis de la variación en la distribución de la pubescencia foliar en 15 ejemplares de Jujuy, Salta y Tucumán.

\begin{tabular}{|c|c|c|}
\hline \multirow{2}{*}{ Colecciones } & \multicolumn{2}{|c|}{ Lámina } \\
\hline & Haz & Envés \\
\hline Chambi y Ramirez 270 & pubescencia esparcida a rala & glabro \\
\hline Garkus 1073 & pubescencia en la mitad inferior & pubescencia glandular \\
\hline Karlsson 62 & glabro & glabro \\
\hline Menendez Sevillano et al. 9333 & pubescente & pubescente en $1 / 2$ inferior \\
\hline Novara 6374 & glabro a subglabro & pubescencia generalizada \\
\hline Novara y Juárez 511 & glabro a subglabro & pubescente \\
\hline Tolaba 746 & pubescencia esparcida a rala & pubescente \\
\hline Tolaba 991 & glabro a subglabro & glandular y con pelos cortos \\
\hline Zapater y Benci 5316 & pubescencia glandular y pelos cortos & pubescencia glandular \\
\hline Zapater y Benci 5517 & pubescencia en la mitad inferior & glandular $1 / 2$ inferior \\
\hline Zapater y Benci 5522 & glabro & pubescencia glandular \\
\hline Zapater y Benci 5584 & glabro & glabro \\
\hline Zapater y Del Castillo 5308 & glabro & pubescencia glandular \\
\hline Zapater y Del Castillo 5534 & glabro a subglabro & pubescencia glandular \\
\hline Zapater y Fuentes 5510 & pubescencia generalizada & pubescente sobre nervio \\
\hline
\end{tabular}


Tabla 2. Localización de las colecciones estudiadas en el análisis de variedades de S. pilifera

\begin{tabular}{ll}
\hline Colecciones & Localidad \\
\hline Chambi y Ramirez 270 & Vaqueros, La Caldera - Salta \\
\hline Garkus 1073 & Campo Quijano, Rosario de Lerma - Salta \\
\hline Karlsson 62 & Río Lorohuasi, Cafayate - Salta \\
\hline Menendez Sevillano et al. 9333 & Alijilán, El Alto - Catamarca \\
\hline Novara 6374 & Gallinato, La Caldera - Salta \\
\hline Novara y Juárez 511 & PN El Rey, Anta - Salta \\
\hline Tolaba 746 & INTA Cerrillos - Salta \\
\hline Tolaba 991 & La Pedrera, Capital - Salta \\
\hline Zapater y Benci 5316 & Acceso a Cafayate - Salta \\
\hline Zapater y Benci 5517 & Rio Chuscha, Cafayate - Salta \\
\hline Zapater y Benci 5522 & Cementerio de la cruz, Capital - Salta \\
\hline Zapater y Benci 5584 & Gallinato, La Caldera - Salta \\
\hline Zapater y Del Castillo 5308 & Acceso a Tapia - Tucumán \\
\hline Zapater y Del Castillo 5534 & Acceso a Guachipas - Salta \\
\hline Zapater y Fuentes 5510 & Campo Militar, Capital - Salta \\
\hline
\end{tabular}

utilizados de glabrescencia o pubescencia en pecíolo, lámina y nervios son confusos y no determinantes a los fines de la diferenciación intra-específica. Estas observaciones concuerdan con lo mencionado por Juárez de Varela y Novara (2007) para la Flora del Valle de Lerma, donde los autores no pudieron diferenciar las variedades piliferus y boliviensis en los materiales estudiados.

En todas las poblaciones estudiadas a campo pudo observarse una clara diferencia morfológica entre los individuos pistilados y estaminados. Los individuos pistilados se caracterizan por abundantes ramas espinescentes largas con nudos numerosos que conforman una copa irregular poco densa versus los individuos estaminados en general con ramas espinescentes cortas y delgadas, con pocos nudos y copa regular, densamente foliosa. Respecto a variaciones morfológicas producto de hábitats diferentes, en general no hay rasgos morfológicos notables que permitan relacionar las plantas con una unidad biogeográfica.

Los caracteres que agregamos y que permiten una segura identificación taxonómica de $S$. pilifera se encuentran en la diagnosis detallada del estudio taxonómico que se presenta previamente y comprende toda la variación de aspectos vegetativos y reproductivos obtenidas de estudios de herbarios y de poblaciones a campo. Se considera de vital importancia para identificar la especie de otras afines, caracteres no considerados previamente como los tricomas glandulares en las hojas y otros órganos y su variación; los caracteres florales del gineceo (ovario, estilo y estigmas); los caracteres del exocarpo del fruto y la forma y capas del endocarpo además de la forma de las hojas de plántulas, plantines y reprotes.

En este sentido las características más relevantes para identificar a $S$. pilifera son: ramas terminales pardas a pardo-rojizas pubescentes con tricomas muy cortos y blancos con densidad variable, a menudo espinescentes; inflorescencias tirsoides con un dicasio sésil por nudo y raquis más o menos piloso; flores pentámeras con pedicelos articulados, gineceo con ovario esférico con pubescencia glandular y de pelos rectos y curvos; estilo corto terminal con tres cabezas estigmáticas grandes; 
fruto esférico morado a negro a la madurez de aproximadamente $0,5 \mathrm{~cm}$ diám., con pilosidad blanca, corta e irregular, mesocarpo ámbar con protuberancias originadas por profundas irregularidades del endocarpo.

\section{Biología floral y reproductiva}

Sistema reproductivo. - La proporción de fruto/flor fue nula en APE en pies estaminados y pistilados, en tanto que en PL fue muy variable de 33,37\% +/- 16,14 en pies pistilados y de $0 \%$ en los estaminados, concordante con plantas diclino-dioicas. El número de granos de polen fue mayor en los estambres del ciclo externo y menor en los del ciclo interno. La planta se caracteriza por tener un óvulo por lo que los valores de P/O's en las flores estaminadas fue muy elevado. En algunas flores se encontraron pocos granos de polen normales en los estaminodios (Tabla 3 y 4).

Tipo de polinización.- Como resultado del embolsamiento con tul de inflorescencias pistiladas, se obtuvieron valores de producción de frutos con un amplio rango entre los cinco pies embolsados, variable entre $15-60 \%$ en promedio según el individuo.

Morfología del fruto.- El fruto definido como una drupa de forma esférica, rosada a rojiza cuando inmadura y negra a la madurez, variablemente pilosa (Fig. 3C, LB), alcanzó en la población estudiada un diámetro de 3,52 +/-0,12; con peso específico de $33,39 \mathrm{~kg} / \mathrm{dm}^{3}$ y peso de 100 frutos con exocarpo de $2,99 \mathrm{~g}$. Visto al MEB, el exocarpo presentó pelos simples y glandulares con diferente grado de densidad; los pelos simples de 0,09-0,18 mm, unicelulares, en su mayoría erectos, algo curvados hacia el ápice, agudos; los glandulares de 0,024-0,027 mm, unicelulares, sésiles. El mesocarpo parenquimático fue de $1,08 \mathrm{~mm}$ de espesor en promedio con seis o más conductos resiníferos grandes y huecos de aproximadamente $0,6 \mathrm{~mm}$ diám., asociados a haces vasculares y recubierto externamente por una capa resinosa color ámbar. El endocarpo resultó profundamente lobulado con crestas de $0,6-0,91 \mathrm{~mm}$, lo que

Tabla 3. Resultados de éxito reproductivo.

\begin{tabular}{|c|c|c|c|}
\hline \multicolumn{2}{|c|}{ Pies con flores pistiladas } & \multicolumn{2}{|c|}{ Pies con flores estaminadas } \\
\hline APA & PL & APA & $\mathrm{PL}$ \\
\hline $0 \%$ & $33,37 \%+/-16,14$ & $0 \%$ & $0 \%$ \\
\hline
\end{tabular}

Tabla 4. Resultados Relación Polen/Óvulos (P/O's).

\begin{tabular}{ccc} 
& Número de granos de polen & \\
\hline Estambres largos & Estambres cortos & Total flor \\
$14000+/-417$ & $8025+/-4307$ & 22025 \\
\hline
\end{tabular}


genera protuberancias notables en el fruto sin exocarpo (Fig. 3D, LB). El endocarpo se observó constituido por tres capas de esclereidas en empalizada: la primera capa externa de oesteoesclereidas de $0,027 \mathrm{~mm}$ de espesor; la siguiente intermedia, muy delgada y constituida por braquiesclereidas con $0,015 \mathrm{~mm}$ y la interna gruesa con macroesclereidas lignificadas de 0,077-0,093 mm de espesor (Fig. 3G, MEB). La semilla se observó con tegumento de espesor variable entre $0,05-0,2 \mathrm{~mm}$, separada del endocarpo (Fig. 3E, LB; Fig. 3F, MEB).

\section{DISCUSIÓN}

Las observaciones realizadas relacionadas con las tres variedades en el sentido de la distribución foliar de la pubescencia, son tan débiles y confusas que no permiten la clara identificación de los taxones, lo que resulta concordante con Juárez de Varela y Novara (2007) para la Flora del Valle de Lerma, donde los autores no pudieron diferenciar las variedades piliferus y boliviensis en los materiales estudiados.

En relación al sistema reproductivo nuestros resultados sugieren que se trata de una especie diclino-dioica, en discordancia con Digilio y Legname (1966) que la mencionan como polígamo-dioica. Se observó formación de frutos y semillas solamente en el tratamiento PL con baja producción, $\mathrm{RR} \%=33,4+/-16,14$ (Tabla 3), lo que podría ser explicado en parte al menos por la presencia de una gruesa membrana estigmática como ocurre en otras especies en las cuales es necesaria la ruptura de la misma por parte de los polinizadores. El número de granos de polen fue muy elevado (Tabla 2.), el doble en los estambres largos respecto a los cortos y teniendo en cuenta los resultados del P/O's, según Cruden (1977) y Dafni (1992), la especie estudiada tiene un sistema xenógamo obligado, dato coincidente con los experimentos a campo y a la característica de ser diclino-dioica.

El bajo éxito reproductivo femenino registrado en el tratamiento de polinización libre, no se condice con la elevada producción de flores en los pies pistilados. La baja producción de frutos en especies dioicas fue mencionada para otras especies en relación a la baja frecuencia de polinizadores o porque estos visitarían más las flores estaminadas que les ofrecen mayor número de recompensas por ser más numerosas y atractivas (Eckhart, 1999; Álvarez, Pérez, Cuevas, 2014). Otra causa podría estar asociada a la perturbación antrópica que se produce por la urbanización creciente de la zona y el alto tránsito vehicular por un camino vecinal a cuya vera se encuentra la población en estudio, como fue analizado en otras especies de Schinus y recientemente en S. areira (Zapater et al., 2018).

La polinización es necesariamente alógama como lo demostró la experiencia de cobertura con voile y los resultados nulos de producción de frutos en APE. La existencia de entomofilia estaría demostrada por características morfológicas florales como la existencia de un disco nectarífero con abundantes estomas secretores, membrana en los estigmas y presentación secundaria del polen en disco. De los experimentos de exclusión de polinizadores con bolsa de tul, resultó que la polinización anemófila es importante y asociada a caracteres florales típicos de este tipo de polinización, como flores unisexuales, expuestas fuera de la masa de las hojas, perianto 
insignificante, anteras y estigmas expuestos y bajo número de óvulos por flor (Faegri y van der Pijl, 1979). Ambas condiciones también fueron recientemente observadas en S. areira (Zapater et al., 2018). Por la presencia de los dos tipos de polinización se concluye que la especie es ambófila, pudiendo en algunos individuos superar la anemofilia (19-60\%) respecto a la entomofilia, en tanto que en otras especies del género es netamente predominante la entomofilia, como en S. longifolia (Lindl.) Speg. (Torreta y Basilio, 2009) y en S. areira (Zapater et al., 2018).

Por otra parte, la presencia de frutos carnosos (drupas) y la instalación de la regeneración de $S$. pilifera en bordes de alambrados, avalaría la hipótesis de Bawa (1980) y Thomson y Brunet (1990), sobre la existencia de una relación entre dioecia, frutos carnosos y dispersión zoocórica. La bibliografía menciona a diversas aves paseriformes como Turdus amaurochalinus, Myopsitta monachus, Pipraeidae bonariensis, Turdus chiguanco, entre otras, como consumidores de drupas en S. areira., S. fasciculatus (Griseb.) I.M. Johnst. y S. longifolius (Lindl.) Speg. (De la Peña y Pensiero, 2017).

$\mathrm{El}$ endocarpo del fruto en $S$. pilifera se correspondió al Grupo A del tipo Anacardium y la Tribu Rhoeae mencionado para $S$. molle L por Copeland (1959), para $S$. terebenthifolia Raddi por Carmello-Guerreiro y Sartori Paoli (2002), y para S. areira por Zapater et al. (2018). Según Baskin et al. (2000), la semilla del Grupo A tipo Anacardium presenta dormición física (DF) por encontrarse el embrión mecánicamente protegido por el endocarpo endurecido compuesto por tres capas de esclereidas en empalizada. La capa interna de macroesclereidas, posee una discontinuidad (el micrópilo carpelar) que permitiría el ingreso de agua al embrión. Las capas más externas de braqui y ostoescrereidas no presentan esta discontinuidad excluyendo así el ingreso del agua al micrópilo carpelar (Baskin et al., 2000). Estas capas externas del endocarpo son entonces las que restringen el ingreso del agua a la semilla.

En sus primeras etapas las plántulas y los plantines o renovales hasta el año de edad, poseen hojas que difieren de las adultas en forma y margen lo que ya fue observado en otras especies de Schinus de hojas simples de la Sect. Pilifera como $S$. johnstonii F. A. Barkley y S. sinuatus (Griseb.) Engl. (Steibel y Troiani, 2008) y mencionado como una característica de la Sección (Silva-Luz et al., 2018). Las hojas de rebrotes de fuste son semejantes a las de plantines con margen aserrado y dientes grandes.

\section{CONCLUSIÓN}

Las poblaciones argentinas de $S$. pilifera pueden delimitarse más ajustadamente respecto a otras especies espinescentes de la Sect. Pilifera, a partir de la diagnosis y otros resultados del estudio taxonómico. Particularmente, el fruto permite una rápida identificación por diferenciarse de otras especies de la Sect. por tamaño, color y pubescencia del exocarpo, además de los caracteres anatómicos.

La nueva circunscripción de esta especie muy polimórfica con la eliminación de las variedades que pasaron a la sinonimia, es concordante con el planteo estructural de la existencia del «complejo Pilifera» sugerido por Silva-Luz et al. (2018). 
El sistema sexual en la población estudiada es diclino-dioico.

El conocimiento de la distribución y el hábitat de las poblaciones de $S$. pilifera es un aporte para la utilización de la especie en la conservación de ambientes naturales para el control de erosión y estabilización de taludes, sobre la base de su asociación a cursos de agua y lugares inundables.

\section{AGRADECIMIENTOS}

Queremos manifestar nuestro profundo agradecimiento a los Curadores y Personal de los Herbarios que consultamos (LIL y MCNS), Dra. Nora Muruaga y Dra. Olga Martínez. También a la Dra. Ana M. González y al Dr. Gabriel Rua por atender nuestras consultas. Este estudio ha sido financiado con el subsidio otorgado al Proyecto de Investigación Tipo A No 2383 por el Consejo de Investigación de la Universidad Nacional de Salta.

\section{BIBLIOGRAFÍA / BIBLIOGRAPHY}

Álvarez, A. M, Pérez, De los Á y Cuevas, E. (2014). Efecto de la fragmentación del hábitat en el éxito reproductivo de Fuchsia parviflora, una especie dioica. Biológicas 16 (1): 30-32.

Barkley, F. A. (1944). Schinus L. Brittonia 5: 160-198.

Barkley, F. A. (1957). Schinus L. Lilloa 28: 4-109.

Baskin, J. M., Baskin, C. C. y Li, X. (2000). Taxonomy, anatomy and evolution of physical dormancy in seeds. Plant Species Biology 15: 139-152.

Bawa, K. S. (1980). Evolution of dioecy in flowering plants. Annual Rewiev of Ecology and Systematics 11: 15-39.

Cabrera, A. L. (1938). Revisión de las Anacardiáceas Austroamericanas. Revista del Museo de La Plata 2: 3-65.

Carmello-Guerreiro, S. M. y Sartori Paoli, A. A. (2002). Ontogeny and structure of the pericarp of Schinus terebinthifolius Raddi (Anacardiaceae). Brazilian Archives of Biology and Technology 45: 73-79.

Copeland, H. F. (1959). The reproductive structures of Schinus molle (Anacardiaceae). Madroño 15: 14-24.

Cruden, R. W. (1977). Pollen-Ovule Ratios: A Conservative Indicator of Breeding Systems in Flowering Plants. Evolution 31: 32-46.

Dafni, A. (1992). Pollination ecology: a practical approach. Oxford, University Press.

D’Ambrogio de Argüeso, A. (1986). Manual de técnicas en histología vegetal. Buenos Aires. Ed. Hemisferio Sur.

De La Peña, M. R. y Pensiero, J. F. (2017). Las plantas como recurso alimenticio de las aves, 1ra. ed. Santa Fe, Ediciones UNL. Libro digital, PDF (Ciencia y Tecnología) Archivo Digital: descarga y online ISBN 978-987-749-078-7

Digilio, A. P. M. y Legname, P. R. (1966). Los árboles indígenas de la provincia de Tu- 
cumán. Opera Lilloana 15: 64-65.

Eckhart, V. M. (1999). Sexual dimorphism in owers and in orescences. En: M. A. Geber, T. E. Dawson, L. F. Delph (Eds.), Gender and sexual dimorphism in flowering plants. Alemania (pp. 123-148). Berlín, Springer-Verlag.

Faegri, K. y Van Der Pijl, L. (1979). The principles of pollination ecology, 3rd edition. Oxford. Pergamon Press.

Johnston, I. M. (1938). Schinus piliferus I.M. Johnston. Fournal Arnold Arb. 19: 256.

Juárez De Varela, F. y Novara, L. J. (2007). Anacardiaceae. En: L. Novara (Ed.), Flora del Valle de Lerma 8(6). Argentina (pp. 1-27). Salta, Aportes Botánicos de Salta, Serie Flora.

Moreno, N. P. (1984). Glosario Botánico Ilustrado. México. Compañía Editorial Continental, S. A. DE C. V.

Muñoz, J. de D. (2000). Anacardiaceae. En: J. Hunziker, A. Anton (Eds.), Flora Fanerogámica Argentina 65: 16-17.

Perea, M. C., Pedraza, G. y Luceros, J. (2007). Relevamiento de la flora arbórea autóctona en la provincia de Catamarca. San Fernando del Valle de Catamarca, Catamarca. 1ra. ed. Ed. Consejo Federal de Inversiones.

Silva-Luz, C. L., Pirani, J. R., Mitchell, J. D., Daly, D., Capelli, N. do V., Demarco, D., Pell, S. K. y Plunkett, G. M. (2018). Phylogeny of Schinus L. (Anacardiaceae) with a new infrageneric classification and insights into evolution of spinescence and floral traits. Molecular Phylogenetics and Evolution, doi: https://doi. org/10.1016/j.ympev.2018.10.013.

Steibel, P. E. y Troiani, H. O. (2008). La identidad de Schinus fasciculatus var. arenicola y rehabilitación de S. sinuatus (Anacardiaceae). Boletín de la Sociedad Argentina de Botánica 43: 157-166.

Torreta, J. P y Basilio, A. M. (2009). Dispersión polínica y éxito reproductivo de cuatro especies arbóreas de un bosque xerófito de Argentina. Revista de Biología Tropical 57: 1-2.

Thomson, J. D. y Brunet, J. (1990). Hypotheses for the evolution of dioecy in seed plants. Tree 5: 11-16.

Zapater, M. A., Alemán, M. M., Lozano, E. C., Aquino, V. H., Flores, C. B., Gil, M. N., Villada, J. y Araya, G. (2018). Aspectos reproductivos de Schinus areira L. (Anacardiaceae). Boletín de la Sociedad. Argentina de Botánica 53 (4): 619-631. 